\title{
Neuroprotective effects of exercise in people with progressive multiple sclerosis (Exercise PRO-MS): study protocol of a phase II trial
}

\author{
A. S. Gravesteijn ${ }^{1 *}$ D, H. Beckerman ${ }^{1}$, B. A. de Jong ${ }^{2}$, H. E. Hulst ${ }^{3}$ and V. de Groot ${ }^{1}$
}

\begin{abstract}
Background: Neurodegeneration, rather than inflammation, plays a key role in the progressive phase of multiple sclerosis (MS). Current disease modifying treatment options for people with progressive MS (PMS) do not specifically target neurodegeneration. Preliminary evidence suggests that exercise therapy might have neuroprotective effects. However, neuroprotective effect studies of exercise interventions in PMS are scarce and the possible mode of action underlying neuroprotective effects of exercise are unknown and need to be elucidated. The main aim of this phase II trial is to assess whether progressive resistance training (PRT) and high intensity interval training (HIIT), can slow down neurodegeneration in people with PMS.

Methods: In a single-blinded phase II clinical trial with an extended baseline period, 60 people with PMS will be randomly assigned to PRT or HIIT. The participants should have had a relapse onset of MS with confirmed disease progression, however still ambulatory. The duration of the study is 48 weeks, consisting of 16 weeks baseline period (no intervention), 16 weeks intervention and 16 weeks follow-up. Patient-tailored training will be performed 3 times per week for one hour in groups, led by an experienced physiotherapist. The primary outcome measure is neurodegeneration, measured as whole brain atrophy on magnetic resonance imaging (MRI). Secondary outcome parameters will include other biomarkers associated with neurodegeneration (i.e. regional brain atrophy, lesion load, white matter integrity, resting state functional connectivity, blood biomarkers (brain derived neurotrophic factor (BDNF) and serum neurofilament light (sNFL)), patient functioning (physical and cognitive) and cardiovascular risk factors.

Discussion: Besides the primary outcome measures, this study will examine a large variety of biomarkers associated with neurodegeneration after an exercise intervention. Combining outcome parameters may help to elucidate the mode of action underlying neuroprotective effects of exercise.
\end{abstract}

Trial registration: This trial is prospectively registered at the Dutch Trial Registry (number NL8265, date 06-01-2020).

Keywords: Progressive multiple sclerosis, Exercise, High intensity interval training, Progressive resistance training, Neurodegeneration

\footnotetext{
* Correspondence: a.gravesteijn@amsterdamumc.nl

${ }^{1}$ Department of Rehabilitation Medicine, MS Center Amsterdam, Amsterdam

Neuroscience research Institute, Amsterdam UMC, Vrije Universiteit

Amsterdam, PO Box 7057, 1007, MB, Amsterdam, the Netherlands

Full list of author information is available at the end of the article
}

(c) The Author(s). 2020 Open Access This article is licensed under a Creative Commons Attribution 4.0 International License, which permits use, sharing, adaptation, distribution and reproduction in any medium or format, as long as you give appropriate credit to the original author(s) and the source, provide a link to the Creative Commons licence, and indicate if changes were made. The images or other third party material in this article are included in the article's Creative Commons licence, unless indicated otherwise in a credit line to the material. If material is not included in the article's Creative Commons licence and your intended use is not permitted by statutory regulation or exceeds the permitted use, you will need to obtain permission directly from the copyright holder. To view a copy of this licence, visit http://creativecommons.org/licenses/by/4.0/ The Creative Commons Public Domain Dedication waiver (http://creativecommons.org/publicdomain/zero/1.0/) applies to the data made available in this article, unless otherwise stated in a credit line to the data. 


\section{Background}

Multiple sclerosis (MS) is a complex disease of the central nervous system (CNS), with inflammatory and neurodegenerative aspects [1]. According to Lublin et al. MS can be categorized in different clinical subtypes, namely active versus not active neuroinflammation, with or without progression [2]. Progressive MS (PMS) is characterized by a gradual deterioration of daily activities due to increasing neurological symptoms [3]. The underlying pathophysiological mechanism for this deterioration is still poorly understood although it is known that neurodegeneration (i.e. axonal and neuronal loss in the CNS) plays a prominent role in PMS $[1,3]$. Neurodegeneration is not yet targeted by the available disease modifying therapies (DMTs). These interventions generally target neuroinflammation $[1,3]$. Some preliminary evidence from animal models, healthy aging individuals and people with MS suggests that exercise might have a neuroprotective effect [4-7].

\section{Exercise and neurodegeneration}

Animal studies on the effects of exercise, demonstrated that endurance training in a mouse model of MS, i.e. experimental autoimmune encephalomyelitis (EAE), resulted in a better structurally preserved nervous system [4]. The endurance exercise group showed less severe motor impairment and autopsy showed less dendritic loss and less synaptic defects, compared to a sedentary mouse group [4].

Also, in healthy people research on the neuroprotective effects of exercise has been conducted. These studies suggest a neuroprotective effect of exercise and physical activity. Endurance training conducted at a moderate-to-vigorous intensity for six months resulted in an increase in grey- and white matter volume, especially in the prefrontal and temporal cortices [5]. Additionally, moderate intensity endurance training can upregulate levels of brain derived neurotrophic factor (BDNF) in healthy aging individuals $[8,9]$. BDNF is a neurotrophin which plays an important role in synaptic plasticity, neuronal survival, differentiation and neuronal growth, and levels of BDNF decrease in neurodegenerative diseases [9-11]. Moreover, BDNF is strongly linked to cognitive functions $[12,13]$.

In people with MS exercise has been widely adopted as an intervention for patient functioning and general well-being. Exercise guidelines recommend twice weekly resistance training and endurance training to improve cardiorespiratory fitness, muscle strength, daily functioning and quality of life [14-16]. Cross-sectional functional MRI data indicate that people with MS who are more physically active (determined as averaged movement counts per day measured with an accelerometer) have increased resting state functional connectivity ( $\mathrm{rSFC}$ ) in the default-mode network (DMN) [17]. rsFC, measured with functional MRI, is a measure of the blood-oxygenlevel-dependent signal used to examine the functional architecture of the brain [18]. The functional architecture of the brain in people with MS generally differs from that of healthy controls, and correlates with MS severity as measured by the Expanded Disability Status Scale (EDSS) [19]. In people with MS decreased rsFC of the DMN is associated with a decreased cognitive functioning $[20,21]$.

A recent systematic review indicated that 2-3 times exercise per week for at least 4 weeks can improve brain function and brain integrity in people with MS [6]. A 24-week progressive resistance training (PRT) intervention study in people with relapsing remitting MS, showed a trend towards brain preservation in the training group. A slowing of the natural loss of brain volume, expressed as percentage brain volume change (PBVC) was found of $-0.01 \%$, (95\% confidence interval $(95 \% \mathrm{CI})$ : -0.15 to 0.13 ) compared to a PBVC of -0.28 , (95\%CI: -0.61 to 0.06$)$ in the waitlist control group [7]. In addition, a better cardiorespiratory fitness is associated with a better integrity of the nervous system and larger (deep) grey matter brain volumes, except for the thalamus [22, 23]. Intervention studies in MS also examined the effect of exercise on rsFC. A 4-week intervention study combining endurance and resistance training demonstrated increased rsFC in the sensorimotor brain network areas [24]. The sensorimotor network plays an important role in motor function and rsFC in the sensorimotor network is decreased in people with MS [25]. Likewise, after a task-oriented walking intervention rsFC increased between sensorimotor areas [26]. An endurance walking intervention in people with MS demonstrated an improved rsFC between thalamic and right superior frontal gyrus, and thalamic and left medial frontal gyrus, associated with better cognitive performance [27]. In addition, white matter microstructural integrity measured with Diffusion Tensor Imaging (DTI) may improve with exercise [28]. Also in MS, an upregulation of BDNF after moderate intensity endurance training has been demonstrated $[6,11,29,30]$.

Exercise is a safe intervention for people with MS and current guidelines recommend exercise and physical activity to people with MS, because of the beneficial heath effect on patient functioning and cardiovascular risk [14, 31]. Exercise might be effective to reduce the cardiovascular risks associated with inactivity $[15,32]$. The above-mentioned studies do indicate that exercise in people with MS has a positive effect on neurodegeneration. However, sample sizes in previous studies were small ( $\mathrm{N}$ varying from 8 to 61), the content, frequency, intensity, and duration of exercise interventions differed, and outcome measures were heterogeneous (brain volume measures, rsFC, DTI- 
parameters, neuronal growth- and neurotrophic factors) [6]. Furthermore, there were no studies that examined the effect of exercise on neurodegeneration specifically in the relapse-onset PMS population. So, at this point the effects of different exercise interventions on neurodegeneration in the PMS population are not known.

\section{Exercise PRO-MS}

In the proposed Exercise PRO-MS study an extensive set of neurodegenerative biomarkers will be used to investigate the effect of two patient-tailored exercise programs, i.e. PRT and high intensity interval training (HIT) on neurodegeneration in people with PMS. Both resistance and endurance training might affect neurodegeneration but currently it is unknown whether one intervention is more beneficial than the other one. In the proposed study we will examine the effectiveness of both interventions on neurodegeneration in the PMS population.

The primary aim of this trial is:

To examine whether PRT and HIIT can slow down neurodegeneration in people with PMS. We expect that participants will benefit from both PRT and HIIT as compared to no training.

Additional objectives of the Exercise PRO-MS study are:

1. To determine whether PRT and HIIT can improve patient functioning in people with PMS.

2. To explore the relationships between patient functioning on the one hand, and biomarkers related to neurodegeneration on the other hand.

3. To determine the relative responsiveness of neurodegeneration outcome measures, and to explore effect sizes of between-intervention comparisons.

4. To determine whether cardiovascular risk factors improve as a consequence of a PRT or a HIIT intervention.

\section{Methods/design}

\section{Design and setting}

A single-blind monocenter randomized phase II trial with extended baseline will be conducted from 2020 till 2023 at the MS Center Amsterdam (MSCA), The Netherlands. MSCA is embedded in the Amsterdam $\mathrm{UMC}$, location VUmc, and is a specialized center for MS care, research and education. In an extended baseline design, participants function as their own control reducing variance and increasing power. During intake participants will be assessed for eligibility for inclusion. In case of inclusion, participants will be scheduled for two baseline measurements sixteen weeks apart. After the second baseline measurement participants will be randomized to either the PRT or HIIT group.
Measurements will be repeated post-intervention and after a 16-week follow-up period.

\section{Randomization and blinding}

Randomization will be performed to prevent selection bias. A computer-generated variable block-randomization model will be used. Block size will be disclosed at the end of the study. Block randomization is applied to achieve an approximately equal group size over time. An independent investigator will inform the physiotherapist about allocation of participants, participants will be notified of the intervention during the first training session. The outcome assessor will be unaware of allocation, and measures will be taken to ensure her blinding during the study. The physiotherapist and participant will not be blinded, since this is not possible.

\section{Study population}

Participants will be recruited through the MS Center Amsterdam, patient organizations and social media. People who are interested will receive a patient information letter. If people are willing to participate an eligibility check will be planned. During this intake visit in- and exclusion criteria will be checked. Prior to all measurements participants will sign the informed consent form. If participants do not meet all inclusion criteria they will be excluded from the study. Inclusion criteria for this study are [1] definite diagnosis of MS according to the 2017 McDonalds criteria, with gradual progression of neurological impairments according to the Lublin criteria 2013 for at least 2 year [2, 33], [2] age between 18 and 70 years, [3] EDSS 3.5-6.0 [34, 35], [4] able to participate in exercise (i.e. no known cardiovascular, pulmonary or metabolic disease, or symptoms of cardiovascular disease), [5] able to understand instructions, and [6] fulfilling MRI safety criteria (no metal inside the body, no claustrophobia and no pregnancy). Exclusion criteria are [1] diagnosis of primary PMS (PPMS), [2] relapse within 3 months before baseline visit, [3] severe comorbidity, objectified as a score $\geq 3$ in 1 or more organ systems on the Cumulative Illness Rating Scale (CIRS) [36], [4] initiation of fampridine within 6 months of baseline, [5] severe depression, objectified as a score $\geq 11$ on the Hospital Anxiety and Depression Scale (HADS) [37], [6] other neurological- and/or musculoskeletal disorders, [7] already participating in a (guided) high intensity exercise training, [8] participating in another intervention study, and [9] pregnancy, given birth in previous 6 months or an active pregnancy wish.

All study procedures will be performed in accordance with the 1964 Helsinki Declaration and its later amendments or comparable ethical standards. Written informed consent will be obtained from all individual participants. 
Withdrawal and replacement of individual participants Participants can leave the study at any time for any reason if they wish to do so without any consequences. The investigator can decide to withdraw a participant from the study for urgent medical reasons. Participants who leave the study prior to random allocation to the exercise programs will be replaced. Participants who leave the study after random allocation will not be replaced. Participants that withdraw from the intervention will be asked to complete assessments post intervention and at follow-up. Also, they will be asked to share the reason for withdrawing from the study (Fig. 1).

\section{Sample size calculation}

Based on pre- and post-intervention measurements, paired analysis will be performed to examine the effect of exercise per type of intervention. Power calculation was performed using data from a previous study examining the effect of a 24-week progressive resistance training (group difference of $0.3 \mathrm{PBVC}$, standard deviation (SD) 0.5, estimated effect size 0.6) [7]. A sample size of 24 achieves $80 \%$ power with a significance level of 0.05 . With an estimated drop-out of $15 \%$ we will include 30 participants per group.

\section{Interventions}

Both interventions (PRT \& HIIT) will be performed three times per week for 16 weeks, supervised by a physiotherapist. Participants can start at different time points. So, group size and composition are variable. Training intensities are based on individual abilities. In case adjustments in exercises or intensities need to be made due to certain disabilities or circumstances, this will be documented and standardized.

\section{Progressive resistance training (PRT)}

The PRT schedule consists of 3 exercises for the lower extremity (leg press, unilateral leg extension and unilateral hamstring curl), 2 exercises for the upper extremity (bench press and seated rows) and 1 core exercise (cable wood chop), to improve muscle strength. Intensity will be at $60-80 \%$ of participants one-repetition maximum (1RM). The training will start with a 5-min warming-up on a bicycle ergometer followed by PRT (Table 1a). The training will end with a cooling down of $5 \mathrm{~min}$ on a bicycle ergometer and $5 \mathrm{~min}$ of stretching and relaxation (see Additional file 1 TIDieR Checklist PRT).

\section{High intensity interval training}

HIIT will be performed on a bicycle ergometer with high intensity interval and rest intervals alternated, to improve the cardiorespiratory fitness. The training will start with a 10-min warm-up on the bicycle on light-tomoderate intensity, then participants will start the HIIT training (Table 1b). The intervention will end with a cool down period of $5 \mathrm{~min}$ bicycling and $5 \mathrm{~min}$ of muscle stretching and relaxation (see Additional file 2 TIDieR Checklist).

During the intervention period and during the 16 weeks pre- and post-intervention participants are asked not to follow an exercise program outside this study, and to keep their level of care constant during this period when possible. Usual medical care is continued, including new treatments when the health situation of the patient changes.

\section{Training intensity}

For the PRT group, to determine muscle strength the $1 \mathrm{RM}$ of every exercise performed during the progressive resistance training will be determined in $\mathrm{kg}$. For the HIIT group, cardiorespiratory fitness will be determined during a cardiopulmonary exercise test (CPET). Outcome measures are peak oxygen consumption $\left(\mathrm{VO}_{2}\right.$ peak) in oxygen per $\mathrm{ml} / \mathrm{min} / \mathrm{kg}$, peak heartrate $(\mathrm{HR}$ peak) in beats per minute and peak load (Wpeak) in Watt. Muscle strength and cardiorespiratory fitness will be re-examined at week 8 of the intervention period, to optimize training intensity (Table 1).

\section{Outcome measures}

Measurement will take place at baseline (week 0), extended baseline (week 16), post-intervention (week 32) and at follow-up (week 48) (Table 2).

\section{Primary outcome measure: percentage brain volume change}

The primary outcome measure of this study is PBVC measured on MRI. A longitudinal assessment of whole brain atrophy will be determined using a high resolution T1weighted image (i.e. three-dimensional magnetizationprepared rapid acquisition with gradient echo). Whole brain atrophy is an overall measure of neurodegeneration in MS $[38,39]$. A valid software tool SIENA (part of FSL, https://fsl.fmrib.ox.ac.uk/fsl/fslwiki/SIENA) is available to determine PBVC automatically [40].

\section{Other neurodegeneration biomarkers}

Imaging The following imaging markers will be determined:

- Grey matter, white matter, subcortical volumes and cortical thickness (as measured with respectively SIENA and FIRST (from fsl) and Freesurfer) [41].

- Lesion load using a fluid-attenuated inversion recovery (FLAIR) image [42].

- White matter integrity measured with DTI [41].

- rsFC will be calculated. In the proposed study we will focus on the rsFC in the sensorimotor network 


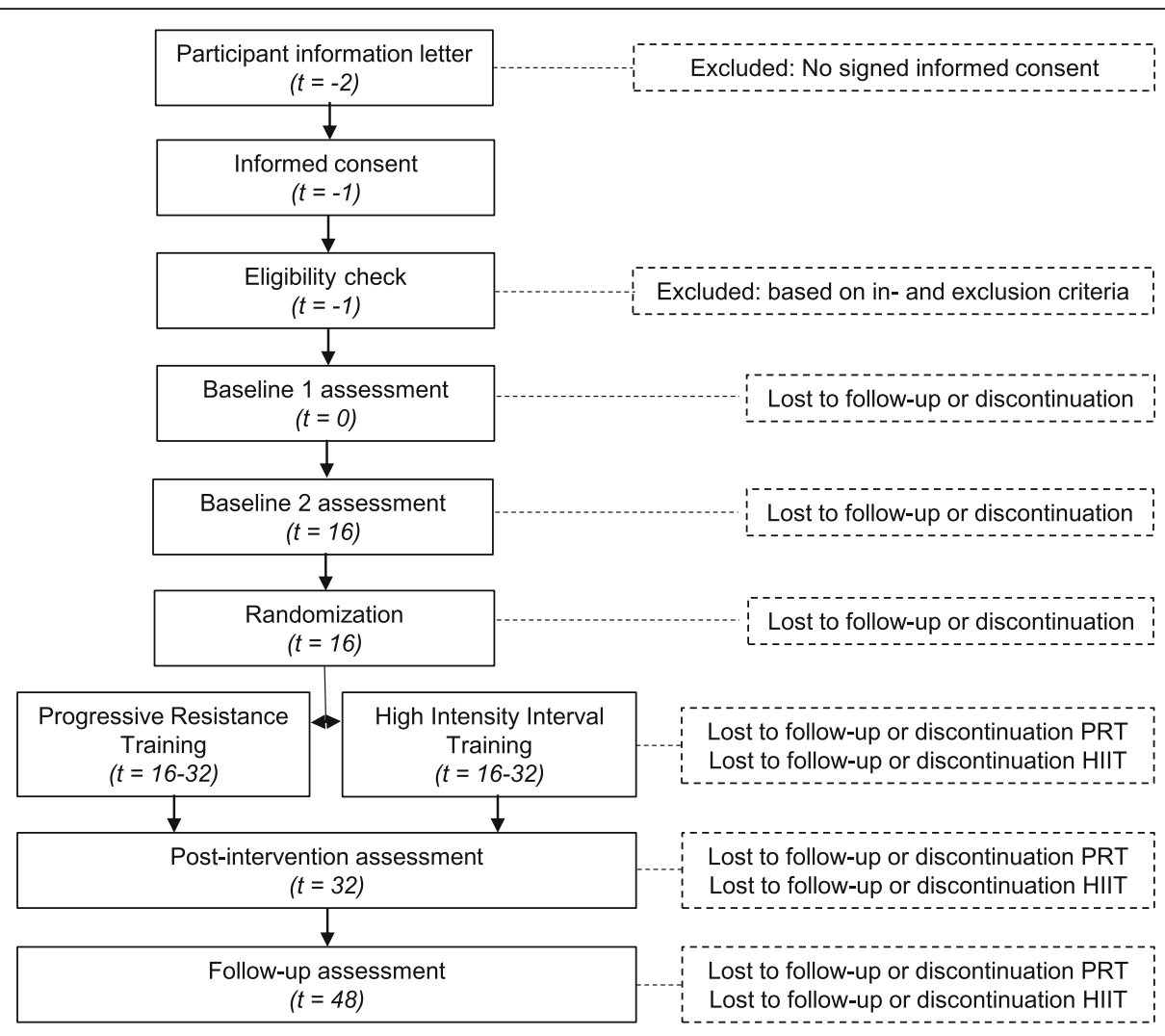

Fig. 1 Participant Timeline

Table 1 Trainings programs, (A) progressive resistance training and (B) high intensity interval training

\begin{tabular}{|c|c|c|c|c|c|c|c|c|}
\hline \multicolumn{4}{|c|}{ A. Progressive Resistance Training } & \multicolumn{5}{|c|}{ B. High Intensity Interval Training } \\
\hline Week & Load (\%1RM) & Volume & Rest (minutes) & Week & Sets & Interval (minutes) & Rest (minutes) & \%peak heartrate \\
\hline \multirow[t]{2}{*}{$1-2$} & 60 & $3 \times 12$ & $2-3$ & 1 & 5 & 1 & 1 & 80 \\
\hline & & & & 2 & 5 & 1 & 2 & 85 \\
\hline \multirow[t]{2}{*}{$3-4$} & 65 & $3 \times 12$ & $2-3$ & 3 & 5 & 1 & 1 & 85 \\
\hline & & & & 4 & 5 & 2 & 2 & 80 \\
\hline \multirow[t]{2}{*}{$5-6$} & 70 & $3 \times 10$ & $2-3$ & 5 & 5 & 2 & 2 & 85 \\
\hline & & & & 6 & 5 & 2 & 2 & 90 \\
\hline 7 & 75 & $4 \times 10$ & $2-3$ & 7 & 4 & 3 & 3 & 85 \\
\hline 8 & \multicolumn{3}{|c|}{ 1RM measurement } & 8 & \multicolumn{4}{|c|}{ Cardiopulmonary exercise test } \\
\hline \multirow[t]{2}{*}{$9-10$} & 65 & $3 \times 12$ & $2-3$ & 9 & 6 & 2 & 2 & 85 \\
\hline & & & & 10 & 4 & 3 & 3 & 80 \\
\hline \multirow[t]{2}{*}{$11-12$} & 70 & $3 \times 10$ & $2-3$ & 11 & 4 & 3 & 3 & 85 \\
\hline & & & & 12 & 4 & 4 & 3 & 80 \\
\hline \multirow[t]{2}{*}{$13-14$} & 75 & $4 \times 10$ & $2-3$ & 13 & 4 & 4 & 3 & 85 \\
\hline & & & & 14 & 4 & 4 & 3 & 90 \\
\hline \multirow[t]{2}{*}{$15-16$} & 80 & $4 \times 10$ & $2-3$ & 15 & 5 & 2 & 2 & 95 \\
\hline & & & & 16 & 5 & 3 & 3 & 90 \\
\hline
\end{tabular}


Table 2 Schedule of enrollment and measurements

\begin{tabular}{|c|c|c|c|c|c|c|}
\hline \multirow[b]{2}{*}{ Week no: } & \multirow{2}{*}{$\begin{array}{l}\text { Enrollment } \\
-1\end{array}$} & \multicolumn{2}{|c|}{ Baseline } & \multirow{2}{*}{$\begin{array}{l}\text { Intervention period } \\
24\end{array}$} & \multicolumn{2}{|c|}{ Post-intervention } \\
\hline & & 0 & 16 & & 32 & 48 \\
\hline Informed consent & $x$ & & & & & \\
\hline Screening & $x$ & & & & & \\
\hline Sociodemographic and clinical characteristics & & $x$ & & & & \\
\hline \multicolumn{7}{|l|}{ Neurodegeneration } \\
\hline Imaging & & $x$ & $x$ & & $x$ & $x$ \\
\hline Blood sampling & & $x$ & $x$ & & $x$ & $x$ \\
\hline \multicolumn{7}{|l|}{ Patient functioning } \\
\hline • Mobility & & $x$ & $x$ & & $x$ & $x$ \\
\hline - Dexterity & & $x$ & $x$ & & $x$ & $x$ \\
\hline • Cognition & & $x$ & $x$ & & $x$ & $x$ \\
\hline - Balance & & $x$ & $x$ & & $x$ & $x$ \\
\hline - Disease & & $x$ & $x$ & & $x$ & $x$ \\
\hline - Psychosocial & & $x$ & $x$ & & $x$ & $x$ \\
\hline - Global perceived effect & & & & & $x$ & $x$ \\
\hline Cardiovascular risk factors & & $x$ & $x$ & & $x$ & $x$ \\
\hline Activity level during follow-up & & & & & & $x$ \\
\hline \multicolumn{7}{|l|}{ Training Intensity } \\
\hline 1RM & & $x$ & $x$ & $x^{a}$ & $x$ & $x$ \\
\hline CPET & & $x$ & $x$ & $x^{a}$ & $x$ & $x$ \\
\hline
\end{tabular}

${ }^{\text {a }}$ Study group dependent

and DMN, which both can be determined reliably in people with MS [43]. Previous exercise intervention studies found changes in rsFC of the sensorimotor network after an exercise intervention [24, 26]. In older healthy individuals also changes in DMN are demonstrated $[44,45]$. In people with MS the rsFC in the DMN is lower compared to healthy controls [20]. In physically active people with MS rsFC in the DMN is higher [17].

Blood sampling Blood biomarkers including BDNF and serum Neurofilament Light (sNFL), will be assessed. Neurofilaments are the major component of axonal cytoskeleton proteins and during axonal damage, neurofilaments are released in the extracellular fluid [46]. sNFL gives an indication of the degree of axonal damage. Beside this, additional blood biomarkers will be determined. The field of research on MS and blood biomarkers is evolving rapidly. Based on these developments it will be determined in a later stage. To determine immunological responses to exercise immune cell profiles will be determined with a combination of flow cytometry by time of flight (CyTOF) mass cytometry and multi-parameter flow cytometry.

\section{Patient functioning}

Mobility Different aspects of walking will be assessed. First, the energy cost of walking will be determined (6MWTec). 6MWTec is a measure of oxygen consumption per meter during walking, which is strongly associated with daily activity and perceived fatigue in MS [47]. Oxygen consumption will be determined during $6 \mathrm{~min}$ of walking with breath-by-breath gas exchange measurements. The last $3 \mathrm{~min}$ of the test are used to determine oxygen consumption, because steady state oxygen consumption is then assumed. Second, the 25-ft timed walk test ( $25 \mathrm{ft}$. TWT) will be performed. Participants will have to perform the $25 \mathrm{ft}$. TWT two times as fast as possible and two times on comfortable walking speed [48, 49]. Third, the patient-reported Multiple Sclerosis Walking Scale (MSWS-12), will be filled in [50].

Dexterity Manual ability of both hands will be measured with the nine-hole peg test (9-HPT) [51].

Cognition The Brief International Cognitive Assessment for MS (BICAMS) will be used to examine information processing speed and immediate verbal and visual recall [52]. The BICAMS consists of the Symbol Digit Modalities Test (SDMT), California Verbal Learning Test-II (CVLT-II) and the Brief Visuospatial Memory Test- 
Revised (BVMT-R). Parallel versions will be used to avoid learning effects. Fifteen healthy controls will also be included and followed over the same 48-week time span to be able to correct for learning effects in the analysis.

Balance The Berg Balance Scale (BBS) will be used to assess balance [53, 54]. In this 14-item scale a rater scores different balance tasks, from sitting without support till standing on one leg, on a 0-4 scale. The total score will be between 0 and 56 , a higher score representing better balance.

Disease severity and impact A disease specific measurement of disability in MS is the EDSS combing impairment(s) in functional systems (pyramidal, cerebellar-, brain stem-, sensory-, bowel \& bladder-, visual-, cerebral-, and other systems) and impairment in walking [34]. Disease impact will be measured with a 29-item patient-reported Multiple Sclerosis Impact Scale (MSIS29) [55].

Psychosocial Depression and anxiety will be assessed with the HADS $[37,56]$. Resilience, the ability to bounce back from challenges occurring in life will be measured with the Connor Davidson Resilience Scale (CDRISC25), a 25-item patient-reported outcome measure [57]. Fatigue will be determined using the Checklist Individual Strength-20r (CIS-20r), a patient-reported questionnaire $[58,59]$. Quality of life will be assessed with the Short-Form health survey-36 (SF-36) [60, 61]. Exercise self-efficacy, confidence in performing exercise will be assessed with the EXercise Self-Efficacy scale (EXSE) $[62,63]$.

Domain specific perceived effect The global changes over a specified time window will be measured using a single question scored on a 7-point scale, also known as the global perceived effect $[64,65]$. Domain specific effect for muscle strength and cardiorespiratory fitness will be determined.

\section{Cardiovascular risk}

People with MS often have a worse cardiovascular risk profile compared to healthy individuals [66]. The unfavorable cardiovascular risk profile might be explained by a sedentary lifestyle common in MS [67]. In people with PMS the cardiovascular risk profile is even worse compared to people with relapse-remitting MS, but this might be explained by a further decline in mobility and daily functioning in the more progressive stages of the disease [68]. Exercise interventions might be beneficial for both patient functioning and cardiovascular risk factors $[69,70]$. Studies do indicate that a higher cardiovascular risk is associated with higher lesion load, more brain atrophy and more disability progression [68, 71, 72]. The following cardiovascular risk factors will be determined; body weight, height, body mass index, \% body fat, blood pressure, High-Density Lipoprotein (HDL), Low Density Lipoprotein (LDL), cholesterol, C-reactive Protein (CRP) and Hemoglobin A1c (HbA1c).

\section{Demographic and clinical characteristics}

Demographic and clinical characteristics will be determined at baseline. The following demographic characteristics will be determined: age, sex, educational level, level of physical activity with the International Physical Activity Questionnaire (IPAQ). The following clinical characteristics will be documented: comorbidities measured with CIRS, year of diagnosis, disease duration, use of medication, use of walking aid, level of education, work and hours of work per week.

\section{Therapy adherence}

Therapy adherence will be monitored. Presence or absence during training sessions will be documented. In the HIIT group the number of performed intervals and intensities will be documented and in the PRT group, the number of repetitions and sets will be documented as well as the weights.

\section{Statistical analysis}

Statistical analysis will be performed using IBM SPSS (Chicago, Illinois) and/or R (Auckland, New Zealand). Nominal data will be presented as frequencies (n) and percentages (\%). Ordinal and interval data will be presented as mean values $(\mathrm{M})$ and SD in case of parametric data and median and range for non-parametric data. A $p$-value $<.05$ will be considered statistically significant for all analyses. To correct for multiple testing the Benjamini-Hochberg procedure will be used [73].

\section{Descriptive statistics}

Demographic and clinical characteristics such as age at first baseline assessment, sex, height, weight, \%body fat, comorbidities with CIRS, medication, years since diagnosis, use of walking aid and EDSS score, will be summarized in a table. For therapy adherence and training intensity, frequencies and percentages will be calculated.

\section{Primary objective}

Within-group paired analysis will be performed for whole brain atrophy measured with PBVC at three different time intervals (difference between baseline 1 and 2 , difference between baseline 2 and post- intervention and difference between post-intervention and follow-up) using linear mixed models The analysis will be 
performed separately for the two different exercise programs. The analysis will be performed using an intention-to-treat method, including all randomized participants, regardless of adherence and measurement completion. Secondary, per protocol analysis will be performed for further exploration of the intervention effects. In addition, one model for the total group of participants with the two different exercise programs as a determinant, will be explored.

\section{Additional objectives}

Longitudinal assessment of change in the different outcome measurements will be determined using a linear mixed model over four different time points. The relationship between (change in) patient functioning measures and (change in) neurodegeneration will be assessed using multiple regression analyses. The relative responsiveness of the various neurodegenerative biomarkers will be analyzed by relating the smallest real difference to the minimally important change of the outcome measures and comparing the area under the receiver operation characteristics curves [74]. Domainspecific patient perceived effect parameters will be used as an anchor. To analyze the effects on cognitive performance, a Reliable Change Index (RCI) will be calculated based on the mean and standard deviation of the healthy controls to adjust for learning effects [75]. An RCI of \pm 1.64 (90\%) will be considered a significant improvement/decline.

\section{Study status}

Currently, participants are being recruited (May 2020).

\section{Discussion}

In PMS neurodegeneration plays a key role in the progression of disability. Up to date there are no DMTs affecting the non-inflammatory drivers of the disease. Previous studies in animals, healthy aging people, and people with MS indicate that exercise training might result in neuroprotective effects and reduced neurodegeneration. However, the interventions used varied, study groups were heterogeneous and sample sizes were most often too small. In this study, the effect of two exercise interventions, PRT and HIIT, will be specifically examined in a homogeneous cohort of people with PMS. Both training interventions are expected to induce neuroprotective effects. Currently it is not known which intervention is more beneficial. In this trial a large variety of neuroprotective outcome measures will be assessed, such as structural and functional imaging parameters of the brain but also biomarkers in blood. In addition, several patient functioning measures will be assessed, for example mobility, dexterity and cognition. Also, the preventive effect of exercise on patient's cardiovascular risk will be studied. The combination of all these outcome measures is one of the major strengths of this study and will hopefully give better insight in the neuroprotective effect of high intensity exercise programs in people with PMS.

In the proposed study we will examine the biomarkers BDNF and SNFL. However, a lot of ongoing research is examining new possible biomarkers for PMS. For example, Glial Fibrillary Acid Protein (GFAP), Vascular Endothelial Growth Factor (VEGF) and sContactin1 and 2. GFAP is a major intermediate cytoskeletal protein of astrocytes and an increase in levels of GFAP is associated with more astrogliosis [76, 77]. VEGF plays a key role in angiogenesis and seems to play a role in neurogenesis $[78,79]$. sContactin 1 and sContactin 2 are potential biomarkers for axonal dysfunction [80]. However, the research on biomarker development is still ongoing and future research might unveil other, more specific neurodegenerative biomarkers. Therefore, participants will be asked to donate some extra blood for possible future analyses.

\section{Supplementary information}

Supplementary information accompanies this paper at https://doi.org/10. 1186/s12883-020-01765-6.

Additional file 1. A document containing the TIDieR checklist for the PRT Intervention.

Additional file 2. A document containing the TIDieR checklist for the HIIT intervention.

\footnotetext{
Abbreviations

1RM: 1-repitition maximum; $25 \mathrm{ft}$. TWT: 25 -ft timed walk test; 6MWTec: 6-min walk test with energy cost measurement; $95 \% \mathrm{Cl}$ : $95 \%$ confidence interval; AE: Adverse event; BBS: Berg Balance Scale; BDNF: Brain derived neurotropic factor; BICAMS: Brief international cognitive assessment for multiple sclerosis; BVMT-R: Brief visuospatial memory test-revised; CD-RISC 25: Connor Davidson resilience scale; CIRS: Cumulative illness rating scale; CIS 20r: Checklist individual strength 20r; CNS: Central nervous system; CPET: Cardiopulmonary exercise test; CRB: Clinical research bureau; CRP: C-reactive protein; CVLTII: California verbal learning test-II; CyTOF: Cytometry by time of flight; DMN: Default-mode network; DMTs: Disease modifying therapies; DTI: Diffusion tensor imaging; EAE: Experimental autoimmune encephalomyelitis; EDSS: Expanded disability status scale; EXSE: Exercise selfefficacy scale; FLAIR: Fluid-attenuated inversion recovery; GCP: Good clinical practice; GFAP: Glial fibrillary acid protein; HADS: Hospital anxiety and depression scale; HbA1c: Hemoglobin A1c; HDL: High-density lipoprotein; HIIT: High intensity interval training; HR peak: Peak heartrate; IPAQ: International physical activity questionnaire; LDL: Low-density lipoprotein; METc: Medical ethical committee; MS: Multiple sclerosis; MSCA: Multiple sclerosis center Amsterdam; MSIS-29: Multiple sclerosis impact scale-29; MSWS-12: Multiple sclerosis walking scale-12; NHPT: Ninehole peg test; PBVC: Percentage brain volume change; PMS: Progressive multiple sclerosis; PPMS: Primary progressive multiple sclerosis; PRT: Progressive resistance training; $\mathrm{RCl}$ : Reliable change index; rsFC: Resting state functional connectivity; SAE: Serious adverse event; SD: Standard deviation; SDMT: Symbol digit modalities test; SF-36: Short -form health survey-36; sNFL: Serum neurofilament light; VEGF: Vascular endothelial growth factors; $\mathrm{VO}_{2}$ peak: Peak oxygen consumption; Wpeak: Peak load in watt
} 


\section{Acknowledgements}

Not applicable.

\section{Monitoring and harms}

An independent monitor from the clinical research bureau (CRB) at Amsterdam UMC, location VUmc will monitor the study according to good clinical practice (GCP). Onsite monitoring will be performed to check informed consent procedure, patient information files, source data verification, conformity of data and (serious) adverse event ((S)AE) reporting Due to the negligible risks of this study, in this study this onsite monitoring is expected to be annually. All (S)AEs during the training period will be documented and all SAE will be reported to the regulatory authorities. The principle investigators and executive investigator are BROK certified. BROK is a mandatory course on regulations and laws in clinical research in The Netherlands.

\section{Ancillary and post-trial care}

Voluntary continuation of training is possible independently of this study. At the end of the training intervention participants receive a letter with information about possibilities to continue training. Participants can receive a copy of their individual training schedule upon request. The Exercise PROMS. study does not provide training interventions outside the intervention studied.

\section{Data collection and management}

Collection of data is subject to the EU general data protection regulation. All data will be stored securely, and the personal information will be coded. The key to this code is only accessible for the principal investigator and involved researchers. Body materials will be stored at Amsterdam UMC, location VUmc for the duration of the study with a participant specific code. Remaining body materials will be destroyed after finalizing the study.

\section{Dissemination policy}

After completion of data collection, the data will be analyzed, and results will be published in scientific journals and presented at (inter)national scientific meetings. Within 9 months after completion of data collection, participants will receive a summary of the results. The identity of the participants will not be disclosed in any of these publications.

\section{Authors' contributions}

VG and $B J$ initiated the study and applied for funding. AG is executive investigator, drafted the manuscript and will perform data collection. VG, HB, $\mathrm{BJ}, \mathrm{HH}$ were involved in the design. $\mathrm{VG}, \mathrm{HB}$ and $\mathrm{AG}$ contributed to the training interventions. $\mathrm{HH}$ designed the MRI protocol. All authors have commented drafts and read and approved the final version of this paper. They all consent publication.

\section{Funding}

This study is funded by the Dutch MS Research Foundation (18-358f MS). The Dutch MS Research Foundation's international scientific advisory committee peer-reviewed the protocol as part of the grant award process. The funder had no influence on the design, and final manuscript.

\section{Availability of data and materials}

Data sharing is not applicable to this article as no datasets were generated or analyzed during the current study. Datasets (generated and/or analyzed) during the proposed study will be available from the corresponding author on reasonable request.

\section{Ethics approval and consent to participate}

This study has been approved by the medical ethical review committee of VU University medical center (METc 2019.676, protocol version 5 date 22-012020 ) and is reviewed and approved by the scientific research committee of Amsterdam Neuroscience research institute (CWO nr.19.27). All amendments, i.e. changes made to the research protocol after a favorable opinion by the METc, will be notified to the METc.

The participant information letter informs about aims, background, measurements, interventions, possible benefits and risks, use of body material (blood sampling), privacy and contact information, including contact information of the independent expert. Before participation participants at least have one week to decide whether to participate based on information in the participant information letter. During this period people have the opportunity to discuss participation with family members, friends, the researcher and an independent expert. Prior to any researchrelated activities, participants must sign the informed consent form. Informed consent will be signed during the first visit by the participant and researcher.

\section{Consent for publication}

Consent for publication of the photographs in Additional file 1 was obtained.

\section{Competing interests}

BJ has received speaker and consulting fees from Merck-Serono, Biogen, TEVA, Genzyme, and Novartis. HH received honoraria for speaking at scientific meetings, serving at scientific advisory boards and consulting activities from, Biogen, Celgene, Genzyme, Merck, and Roche. She has received research support from the Dutch MS Research foundation and serves on the editorial board of MSJ. VG, HB, AG declare to have no competing interests.

\section{Author details}

'Department of Rehabilitation Medicine, MS Center Amsterdam, Amsterdam Neuroscience research Institute, Amsterdam UMC, Vrije Universiteit Amsterdam, PO Box 7057, 1007, MB, Amsterdam, the Netherlands. ${ }^{2}$ Department of Neurology, MS Center Amsterdam, Amsterdam Neuroscience research Institute, Amsterdam UMC, Vrije Universiteit Amsterdam, PO Box 7057, 1007, MB, Amsterdam, the Netherlands. ${ }^{3}$ Department of Anatomy and Neurosciences, MS Center Amsterdam, Amsterdam Neuroscience research Institute, Amsterdam UMC, Vrije Universiteit Amsterdam, PO Box 7057, 1007, $\mathrm{MB}$, Amsterdam, the Netherlands.

Received: 1 April 2020 Accepted: 4 May 2020

Published online: 11 May 2020

\section{References}

1. Thompson AJ, Baranzini SE, Geurts J, Hemmer B, Ciccarelli O. Multiple sclerosis. Lancet. 2018;391(10130):1622-36.

2. Lublin FD, Reingold SC, Cohen JA, Cutter GR, Sorensen PS, Thompson AJ, et al. Defining the clinical course of multiple sclerosis: the 2013 revisions. Neurology. 2014;83(3):278-86.

3. Ontaneda D, Fox RJ. Progressive multiple sclerosis. Curr Opin Neurol. 2015; 28(3):237-43.

4. Rossi S, Furlan R, De Chiara V, Musella A, Lo Giudice T, Mataluni G, et al. Exercise attenuates the clinical, synaptic and dendritic abnormalities of experimental autoimmune encephalomyelitis. Neurobiol Dis. 2009;36(1):51-9.

5. Colcombe SJ, Erickson KI, Scalf PE, Kim JS, Prakash R, McAuley E, et al. Aerobic exercise training increases brain volume in aging humans. J Gerontol Ser A Biol Sci Med Sci. 2006;61(11):1166-70.

6. Negaresh R, Motl RW, Zimmer P, Mokhtarzade M, Baker JS. Effects of exercise training on multiple sclerosis biomarkers of central nervous system and disease status: a systematic review of intervention studies. Eur J Neurol. 2019;26(5):711-21.

7. Kjølhede T, Siemonsen S, Wenzel D, Stellmann J-P, Ringgaard S, Pedersen $B G$, et al. Can resistance training impact MRI outcomes in relapsingremitting multiple sclerosis? Mult Scler J. 2018;24(10):1356-65.

8. Håkansson K, Ledreux A, Daffner K, Terjestam Y, Bergman P, Carlsson R, et al. BDNF responses in healthy older persons to 35 minutes of physical exercise, cognitive training, and mindfulness: associations with working memory function. J Alzheimers Dis. 2016;55(2):645-57.

9. de Coelho FGM, Gobbi S, Andreatto CAA, Corazza DI, Pedroso RV, SantosGalduróz RF. Physical exercise modulates peripheral levels of brain-derived neurotrophic factor (BDNF): a systematic review of experimental studies in the elderly. Arch Gerontol Geriatr. 2013;56(1):10-5.

10. Wang R, Holsinger RMD. Exercise-induced brain-derived neurotrophic factor expression: therapeutic implications for Alzheimer's dementia. Ageing Res Rev. 2018;48(September):109-21.

11. Mackay CP, Kuys SS, Brauer SG. The effect of aerobic exercise on brainderived neurotrophic factor in people with neurological disorders: a systematic review and meta-analysis. Neural Plast. 2017;2017:1-9.

12. White $L$, Castellano V. Exercise and brain health - implications for multiple sclerosis: part 1 - neuronal growth factors. Sports Med. 2008;38(2):91-100.

13. Weinstock-Guttman B, Zivadinov R, Tamaño-Blanco M, Abdelrahman N, Badgett D, Durfee J, et al. Immune cell BDNF secretion is associated with 
white matter volume in multiple sclerosis. J Neuroimmunol. 2007 Aug; 188(1-2):167-74.

14. Latimer-Cheung AE, Martin Ginis KA, Hicks AL, Motl RW, Pilutti LA, Duggan $M$, et al. Development of evidence-informed physical activity guidelines for adults with multiple sclerosis. Arch Phys Med Rehabil. 2013;94(9):1829-1836.e7.

15. Warburton DER. Health benefits of physical activity: the evidence. Can Med Assoc J. 2006;174(6):801-9.

16. Dalgas U, Stenager E, Jakobsen J, Petersen T, Hansen HJ, Knudsen C, et al. Resistance training improves muscle strength and functional capacity in multiple sclerosis. Neurology. 2009;73(18):1478-84.

17. Prakash RS, Patterson B, Janssen A, Abduljalil A, Boster A. Physical activity associated with increased resting-state functional connectivity in multiple sclerosis. J Int Neuropsychol Soc. 2011;17(6):986-97.

18. Biswal B, Zerrin Yetkin F, Haughton VM, Hyde JS. Functional connectivity in the motor cortex of resting human brain using echo-planar mri. Magn Reson Med. 1995;34(4):537-41.

19. Rocca MA, Valsasina P, Leavitt VM, Rodegher M, Radaelli M, Riccitelli GC, et al. Functional network connectivity abnormalities in multiple sclerosis: correlations with disability and cognitive impairment. Mult Scler J. 2018; 24(4):459-71.

20. Roosendaal SD, Hulst HE, Vrenken H, Feenstra HEM, Castelijns JA, Pouwels PJW, et al. Structural and functional hippocampal changes in multiple sclerosis patients with intact memory function. Radiology. 2010;255(2):595-604.

21. Rocca MA, Valsasina P, Absinta M, Riccitelli G, Rodegher ME, Misci P, et al. Default-mode network dysfunction and cognitive impairment in progressive MS. Neurology. 2010;74(16):1252-9.

22. Motl RW, Pilutti LA, Hubbard EA, Wetter NC, Sosnoff JJ, Sutton BP. Cardiorespiratory fitness and its association with thalamic, hippocampal, and basal ganglia volumes in multiple sclerosis. Neurolmage Clin. 2015;7:661-6.

23. Prakash RS, Snook EM, Motl RW, Kramer AF. Aerobic fitness is associated with gray matter volume and white matter integrity in multiple sclerosis. Brain Res. 2010;1341:41-51.

24. Tavazzi E, Bergsland N, Cattaneo D, Gervasoni E, Laganà MM, Dipasquale O, et al. Effects of motor rehabilitation on mobility and brain plasticity in multiple sclerosis: a structural and functional MRI study. J Neurol. 2018; 265(6):1393-401.

25. Janssen AL, Boster A, Patterson BA, Abduljalil A, Prakash RS. Resting-state functional connectivity in multiple sclerosis: an examination of group differences and individual differences. Neuropsychologia. 2013;51(13):2918-29.

26. Fling BW, Martini DN, Zeeboer E, Hildebrand A, Cameron M. Neuroplasticity of the sensorimotor neural network associated with walking aid training in people with multiple sclerosis. Mult Scler Relat Disord. 2019;31(March):1-4.

27. Sandroff BM, Wylie GR, Sutton BP, Johnson CL, DeLuca J, Motl RW. Treadmill walking exercise training and brain function in multiple sclerosis: preliminary evidence setting the stage for a network-based approach to rehabilitation. Mult Scler J - Exp Transl Clin. 2018;4(1):205521731876064.

28. Ibrahim I, Tintera J, Skoch A, Jirů F, Hlustik P, Martinkova P, et al. Fractional anisotropy and mean diffusivity in the corpus callosum of patients with multiple sclerosis: the effect of physiotherapy. Neuroradiology. 2011;53(11): 917-26.

29. Ozkul C, Guclu-Gunduz A, Irkec C, Fidan I, Aydin Y, Ozkan T, et al. Effect of combined exercise training on serum brain-derived neurotrophic factor, suppressors of cytokine signaling 1 and 3 in patients with multiple sclerosis. J Neuroimmunol. 2018;316(January):121-9.

30. Wens I, Keytsman C, Deckx N, Cools N, Dalgas U, Eijnde BO. Brain derived neurotrophic factor in multiple sclerosis: effect of 24 weeks endurance and resistance training. Eur J Neurol. 2016;23(6):1028-35.

31. Pilutti LA, Platta ME, Motl RW, Latimer-Cheung AE. The safety of exercise training in multiple sclerosis: a systematic review. J Neurol Sci. 2014;343(12):3-7.

32. Hallal PC, Bauman AE, Heath GW, Kohl HW, Lee I-M, Pratt M. Physical activity: more of the same is not enough. Lancet. 2012;380(9838):190-1.

33. Thompson AJ, Banwell BL, Barkhof F, Carroll WM, Coetzee T, Comi G, et al. Diagnosis of multiple sclerosis: 2017 revisions of the McDonald criteria. Lancet Neurol. 2018;17(2):162-73.

34. Kurzke JF. Rating neurologic impairment in multiple sclerosis: an expanded disability status scale (EDSS). Neurology. 1983;33:1444-52.

35. Meyer-Moock S, Feng YS, Maeurer M, Dippel FW, Kohlmann T. Systematic literature review and validity evaluation of the expanded disability status scale (EDSS) and the multiple sclerosis functional composite (MSFC) in patients with multiple sclerosis. BMC Neurol. 2014;14(1):1-10.
36. de Groot V, Beckerman H, Lankhorst GJ, Bouter LM. How to measure comorbidity. A critical review of available methods. J Clin Epidemiol. 2003; 56(3):221-9

37. Zigmond AS, Snaith RP. The hospital anxiety and depression scale. Acta Psychiatr Scand. 1983;67(6):361-70.

38. Ontaneda D, Fox RJ. Imaging as an outcome measure in multiple sclerosis. Neurotherapeutics. 2017;14(1):24-34.

39. Wattjes MP, Rovira A, Miller D, Yousry TA, Sormani MP, De Stefano N, et al. Evidence-based guidelines: MAGNIMS consensus guidelines on the use of MRI in multiple sclerosis - establishing disease prognosis and monitoring patients. Nat Rev Neurol. 2015;11(10):597-606.

40. Rocca MA, Battaglini M, Benedict RHB, De Stefano N, Geurts JJG, Henry RG, et al. Brain MRI atrophy quantification in MS: from methods to clinical application. Neurology. 2017:88(4):403-13.

41. Barkhof F, Calabresi PA, Miller DH, Reingold SC. Imaging outcomes for neuroprotection and repair in multiple sclerosis trials. Nat Rev Neurol. 2009; 5(5):256-66.

42. Gramsch C, Nensa F, Kastrup O, Maderwald S, Deuschl C, Ringelstein A, et al Diagnostic value of 3D fluid attenuated inversion recovery sequence in multiple sclerosis. Acta Radiol. 2015;56(5):622-7.

43. Pinter D, Beckmann C, Koini M, Pirker E, Filippini N, Pichler A, et al. Reproducibility of resting state connectivity in patients with stable multiple sclerosis. PLoS One. 2016;11(3):1-11.

44. McGregor KM, Crosson B, Krishnamurthy LC, Krishnamurthy V, Hortman K, Gopinath K, et al. Effects of a 12-week aerobic spin intervention on resting state networks in previously sedentary older adults. Front Psychol. 2018; 9(NOV):1-13.

45. Voss MW, Prakash RS, Erickson KI, Basak C, Chaddock L, Kim JS, et al. Plasticity of brain networks in a randomized intervention trial of exercise training in older adults. Front Aging Neurosci. 2010;2(August):1-17.

46. Teunissen CE, Khalil M. Neurofilaments as biomarkers in multiple sclerosis. Mult Scler J. 2012;18(5):552-6.

47. Motl RW, Sandroff BM, Suh Y, Sosnoff JJ. Energy cost of walking and its association with gait parameters, daily activity, and fatigue in persons with mild multiple sclerosis. Neurorehabil Neural Repair. 2012;26(8):1015-21.

48. Motl RW, Cohen JA, Benedict R, Phillips G, LaRocca N, Hudson LD, et al. Validity of the timed 25-foot walk as an ambulatory performance outcome measure for multiple sclerosis. Ther Adv Vaccines. 2017;23(5):704-10.

49. Decavel P, Moulin T, Sagawa Y. Gait tests in multiple sclerosis: reliability and cut-off values. Gait Posture. 2019;67(February 2018):37-42.

50. McGuigan C, Hutchinson M. The multiple sclerosis impact scale (MSIS-29) is a reliable and sensitive measure. J Neurol Neurosurg Psychiatry. 2004;75(2): 266-9.

51. Feys P, Lamers I, Francis G, Benedict R, Phillips G, LaRocca N, et al. The ninehole peg test as a manual dexterity performance measure for multiple sclerosis. Mult Scler J. 2017;23(5):711-20.

52. Corfield F, Langdon D. A systematic review and meta-analysis of the brief cognitive assessment for multiple sclerosis (BICAMS). Neurol Ther. 2018;7(2): 287-306.

53. Cattaneo D, Jonsdottir J, Repetti S. Reliability of four scales on balance disorders in persons with multiple sclerosis. Disabil Rehabil. 2007;29(24): 1920-5.

54. Cattaneo D, Regola A, Meotti M. Validity of six balance disorders scales in persons with multiple sclerosis. Disabil Rehabil. 2006;28(12):789-95.

55. Hobart J, Lamping D, Fitzpartick R, Riazi A, Thompson A. The multiple sclerosis impact scale (MSIS-29): a new patient-based outcome measure. Brain. 2002;124(5):962-73.

56. Honarmand K, Feinstein A. Validation of the hospital anxiety and depression scale for use with multiple sclerosis patients. Mult Scler. 2009;15(12):1518-24.

57. Connor KM, Davidson JRT. Development of a new resilience scale: the Connor-Davidson resilience scale (CD-RISC). Depress Anxiety. 2003;18(2):76-82.

58. Worm-Smeitink M, Gielissen M, Bloot L, van Laarhoven HWM, van Engelen $B G M$, van Riel P, et al. The assessment of fatigue: psychometric qualities and norms for the checklist individual strength. J Psychosom Res. 2017;98(May): 40-6.

59. Rietberg MB, Van Wegen EEH, Kwakkel G. Measuring fatigue in patients with multiple sclerosis: reproducibility, responsiveness and concurrent validity of three Dutch self-report questionnaires. Disabil Rehabil. 2010;32(22):1870-6.

60. Ware JE, Donald Sherbourne C. The MOS 36-itme short-form health survey (SF-36): I. conceptual framework and item selection. Med Care. 1992;30(6): 473-83. 
61. Riazi A, Hobart JC, Lamping DL, Fitzpatrick R, Thompson AJ. Evidence-based measurement in multiple sclerosis: the psychometric properties of the physical and psychological dimensions of three quality of life rating scales. Mult Scler. 2003;9(4):411-9.

62. McAuley E. Self-efficacy and the maintenance of exercise participation in older adults. J Behav Med. 1993;16(1):103-13.

63. Motl RW, Snook EM, McAuley E, Scott JA, Douglass ML. Correlates of physical activity among individuals with multiple sclerosis. Ann Behav Med. 2006;32(2):154-61.

64. Hudak PL, Wright JG. The characteristics of patient satisfaction measures. Spine (Phila Pa 1976). 2000;25(24):3167-77.

65. Kamper S. Global rating of change scales. Aust J Physiother. 2009;55(4):289

66. Wens I, Dalgas U, Stenager E, Eijnde BO. Risk factors related to cardiovascular diseases and the metabolic syndrome in multiple sclerosis - a systematic review. Mult Scler J. 2013:19(12):1556-64.

67. Ellis T, Motl RW. Physical activity behavior change in persons with neurologic disorders: overview and examples from Parkinson disease and multiple sclerosis. J Neurol Phys Ther. 2013;37(2):85-90.

68. Moccia M, Lanzillo R, Palladino R, Maniscalco GT, De Rosa A, Russo C, et al. The Framingham cardiovascular risk score in multiple sclerosis. Eur J Neurol. 2015;22(8):1176-83.

69. Jorissen W, Vanmierlo T, Wens I, Somers V, Van Wijmeersch B, Bogie JF, et al. Twelve weeks of medium-intensity exercise therapy affects the lipoprotein profile of multiple sclerosis patients. Int J Mol Sci. 2018:19(1):12-21.

70. Keytsman C, Hansen D, Wens I. O. Eijnde B. impact of high-intensity concurrent training on cardiovascular risk factors in persons with multiple sclerosis-pilot study. Disabil Rehabil. 2019;41(4):430-5.

71. Weinstock-Guttman B, Zivadinov R, Mahfooz N, Carl E, Drake A, Schneider J, et al. Serum lipid profiles are associated with disability and MRI outcomes in multiple sclerosis. J Neuroinflammation. 2011;8:1-7.

72. Kappus N, Weinstock-Guttman B, Hagemeier J, Kennedy C, Melia R, Carl E, et al. Cardiovascular risk factors are associated with increased lesion burden and brain atrophy in multiple sclerosis. J Neurol Neurosurg Psychiatry. 2016; 87(2):181-7.

73. Benjamini Y, Hochberg Y. Controlling the false discovery rate: a practical and powerful approach to multiple testing. J R I State Dent Soc. 1995;57(1): 289-300.

74. De Groot V, Beckerman H, Uitdehaag BMJ, De Vet HCW, Lankhorst GJ, Polman $\mathrm{CH}$, et al. The usefulness of evaluative outcome measures in patients with multiple sclerosis. Brain. 2006;129(10):2648-59.

75. Jacobson NS, Truax P. Clinical significance: a statistical approach to defining meaningful change in psychotherapy research. J Consult Clin Psychol. 1991; 59(1):12-9.

76. Högel H, Rissanen E, Barro C, Matilainen M, Nylund M, Kuhle J, et al. Serum glial fibrillary acidic protein correlates with multiple sclerosis disease severity. Mult Scler J. 2020;26(2):210-9.

77. Abdelhak A, Huss A, Kassubek J, Tumani H, Otto M. Serum GFAP as a biomarker for disease severity in multiple sclerosis. Sci Rep. 2018;8(1):14798 Erratum in: Sci Rep. 2019;9(1):9-10.

78. De Almodovar CR, Lambrechts D, Mazzone M, Carmeliet P. Role and therapeutic potential of VEGF in the nervous system. Physiol Rev. 2009;89(2): 607-48.

79. Lin W. Neuroprotective effects of vascular endothelial growth factor a in the experimental autoimmune encephalomyelitis model of multiple sclerosis. Neural Regen Res. 2017;12(1):70-1.

80. Chatterjee M, Koel-Simmelink MJ, Verberk IM, Killestein J, Vrenken H, Enzinger C, et al. Contactin-1 and contactin-2 in cerebrospinal fluid as potential biomarkers for axonal domain dysfunction in multiple sclerosis. Mult Scler J - Exp Transl Clin. 2018;4(4):205521731881953.

\section{Publisher's Note}

Springer Nature remains neutral with regard to jurisdictional claims in published maps and institutional affiliations.

Ready to submit your research? Choose BMC and benefit from:

- fast, convenient online submission

- thorough peer review by experienced researchers in your field

- rapid publication on acceptance

- support for research data, including large and complex data types

- gold Open Access which fosters wider collaboration and increased citations

- maximum visibility for your research: over $100 \mathrm{M}$ website views per year

At $\mathrm{BMC}$, research is always in progress.

Learn more biomedcentral.com/submissions 